\title{
Electron weak localization in disordered films
}

\author{
Tan Li and Ping Sheng \\ Department of Physics, The Hong Kong University of Science and Technology, Clear Water Bay, Kowloon, Hong Kong
}

(Received 20 February 1996)

\begin{abstract}
The logarithmic temperature dependence of resistivity, commonly observed in disordered films, has generally been interpreted as evidence for electron weak localization, with its slope indicative of the inelastic scattering mechanism. In this work, we show that the two-dimensional (2D) quantum percolation (QP) model, pertaining to disordered metallic films, predicts a sample-size-dependent $\ln L$ conductance correction that is three times larger than that for the Anderson model. Moreover, when the film has a finite thickness, the coefficient of $\ln L$ decreases to about $2 / 3$ of its $2 \mathrm{D}$ value for both the QP and the Anderson models. These results have direct implications for the interpretation of experimental data. [S0163-1829(96)51220-8]
\end{abstract}

\section{INTRODUCTION}

The logarithmic temperature dependence of the resistivity, widely observed in disordered films at temperatures below $10 \mathrm{~K}$, is generally regarded as evidence for the weak localization of electrons, arising from the coherent backscattering correction to the film conductance. ${ }^{1-4}$ For twodimensional (2D) samples, this correction term has the form $\alpha\left(e^{2} / \pi^{2} \hbar\right) \ln L$ at $T=0$, where $L$ denotes the sample size, $e$ is the electronic charge, $\hbar$ is Planck's constant, and $\alpha$ is usually taken as a "universal" constant independent of the amount of randomness and other details of the model. One can deduce from the perturbative calculation in the weak scattering limit ${ }^{5}$ that $\alpha=1$ for the Anderson model. To link this sample-size dependence to the temperature dependence, it is recognized that, at finite temperatures, the sample size is replaced by the dephasing length $L_{\mathrm{ph}}$ whenever $L>L_{\mathrm{ph}}$. Since $L_{\mathrm{ph}}$ is related to the inelastic scattering length and is therefore proportional to $T^{-p / 2}$, where the value of $p$ depends on the nature of the inelastic scattering mechanism, it immediately follows that the finite-temperature manifestation of the coherent backscattering correction has the form $\alpha p\left(e^{2} / 2 \pi^{2} \hbar\right) \ln T$. From the experimentally measured slope of the $\ln T$ variation and the magnitude of the resistivity, ${ }^{6,7}$ it is possible to deduce the value of the product $\alpha p$ for various material systems. Based on the assumption that $\alpha$ is known, the value of $p$, and thus the nature of the dominant inelastic scattering mechanism of the system, may be determined.

In this work, we show that while the value of $\alpha$ is indeed independent of the amount of randomness, it is nevertheless nonuniversal. In particular, for the quantum percolation (QP) model $\alpha$ is about three times that of the Anderson model. Furthermore, our numerical results also indicate that for both the QP model and the Anderson model the value $\alpha$ for finitethickness films is about $2 / 3$ of its $2 \mathrm{D}$ value. This is the case when there is scattering in the vertical direction, so that the coherent backscattering correction is reduced. Since for disordered metallic films the QP model is more realistic than the Anderson model, which pertains to doped semiconductors, it follows that many prior experimental results on metallic films may have to be reinterpreted in regard to their implications about the inelastic scattering mechanism(s).

\section{MODELS AND NUMERICAL APPROACH}

For both the QP model and the Anderson model, the Hamiltonian is defined as

$$
H=\sum_{i} \epsilon_{i}|i\rangle\left\langle i\left|+t \sum_{[i j]}\right| i\right\rangle\langle j|
$$

Here $t$ is the nearest-neighbor hopping matrix element, taken to be 1 in this work, and $[i j]$ denotes the nearest-neighbor site indices of a square (cubic) lattice. For the QP model,

$$
\epsilon_{i}= \begin{cases}0 & \text { if site } i \text { is occupied by a metal particle } \\ \infty & \text { if site } i \text { is occupied by an insulator. }\end{cases}
$$

We also define $P$ to be the probability that a site is occupied by a metal particle. In contrast, the Anderson model is defined by assigning $\epsilon_{i}$ to be a random number with a flat distribution extending from $-W / 2$ to $W / 2$.

For percolating metallic films with $P$ close to 1 , conduction electrons have a single Fermi energy throughout the connected metallic network. Also, electrons cannot penetrate into the insulator. Hence, the QP model is clearly descriptive of metallic films when $P$ is close to 1 . In contrast, there is a dispersion of electronic energy levels in disordered semiconductors, so the Anderson model is more descriptive.

To calculate the conductance $G$ of the sample described by either the QP model or the Anderson model, two sides of the disordered $L \times L \times M$ samples with $L \gg M$ are connected to perfect leads, i.e., $\epsilon_{i}=0$ for all the sites in the lead, and the Landauer-Büttiker formula ${ }^{1}$ is used, i.e.,

$$
\gamma=\frac{G}{2 e^{2} / h}=\left(\sum_{i}^{N} T_{i}\right)\left(\frac{2 \sum_{i}^{N} v_{i}^{-1}}{\sum_{i}^{N}\left(1+R_{i}-T_{i}\right) v_{i}^{-1}}\right)
$$

where $N$ is the number of propagating channels in the leads. The values of $T_{i}$ and $R_{i}$ are related to the transmission and reflection matrices by 


$$
T_{i}=\sum_{j=1}^{N}\left|t_{i j}\right|^{2} ; \quad R_{i}=\sum_{j=1}^{N}\left|r_{i j}\right|^{2},
$$

which in turn can be related to the Green's function by ${ }^{3}$

$$
\left|t_{i, j}\right|^{2}=\left|v_{i} v_{j}\right|\left|G_{i, j}^{+}(0, L+1)\right|^{2}
$$

and

$$
\left|r_{i, j}\right|^{2}=\left|i \sqrt{v_{i} v_{j}} G_{i, j}^{+}(0,0)-\delta_{i, j}\right|^{2} .
$$

Here $G_{i, j}^{+}\left(n, n^{\prime}\right)$ is the retarded Green's function with source at $n^{\prime}$ of channel $j$ and receiver at $n$ of the channel $i$. The positions 0 and $L+1$ are located inside the sample on the incoming side and outside the sample on the outgoing lead, respectively. $G_{i, j}^{+}\left(n, n^{\prime}\right)$ can be calculated numerically by use of a recursive technique. ${ }^{2} \mathrm{~A}$ hard wall boundary condition was chosen along the transverse direction(s). The Hamiltonian of the lead $H_{\text {lead }}$ can be written as

$$
H_{\text {lead }}=H_{x} \oplus H_{\perp},
$$

where $H_{x}$ and $H_{\perp}$ are the Hamiltonians for hopping along the $x$ direction and the transverse direction(s), respectively. The eigenfunctions and the corresponding eigenenergies of $H_{x}$ are $\exp \left(i k_{x} a x\right)$ and $2 \cos k_{x} a$, respectively, where $a$ is the lattice constant. Furthermore, we call $E_{\perp}$ the eigenenergy of $H_{\perp}$, the eigenfunctions of which define the channels. Hence, the real solutions $k_{x}$ in the dispersion relation

$$
E=2 \cos k_{x} a+E_{\perp}
$$

determine the number of allowed propagating channels $N$. The channel velocity $v_{i}$ in Eqs. (1), (4), and (5) is given by

$$
v_{i}=\left.\frac{\partial E}{\partial k_{x}}\right|_{k_{x}(i)}=\sqrt{4-\left[E-E_{\perp}(i)\right]^{2}} .
$$

In our calculations the Fermi energy is fixed at $E=0.01$. $^{4}$

\section{RESULTS AND DISCUSSION}

Numerical calculations for 2D $(M=1)$ samples based on the QP model and the Anderson model are shown in Figs. 1(a) and 1(b), respectively. 1000-3000 configurations were used in the average. The straight lines in each figure represent the least-squares fits of

$$
\gamma=\gamma_{0}-\beta \ln L
$$

to the data calculated by Eq. (2), where $\beta$ is related to $\alpha$ by $\alpha=\beta \pi$. Figures 2(a) and 2(b) also show the numerical results for multilayer samples based on the two models. In all cases, the thickness $M$ is always much smaller than the localization length. Therefore these systems are quasi-2D in nature, and we still expect the $\ln L$ sample-size dependence to prevail. The values of $\beta$ and $\alpha$ are tabulated in Tables I and II.

Our results clearly show that the value of $\alpha$ for the QP model is indeed independent of $P$, at least when $P$ is close to 1. This is analogous to $\alpha$ 's independence from $W$ in the Anderson model [see Figs. 1(b) and 2(b)] as demonstrated by the weak-localization theory. ${ }^{5} \mathrm{We}$ found from the leastsquares fits of Eq. (9) to the data that $\alpha=3$ for the 2D QP
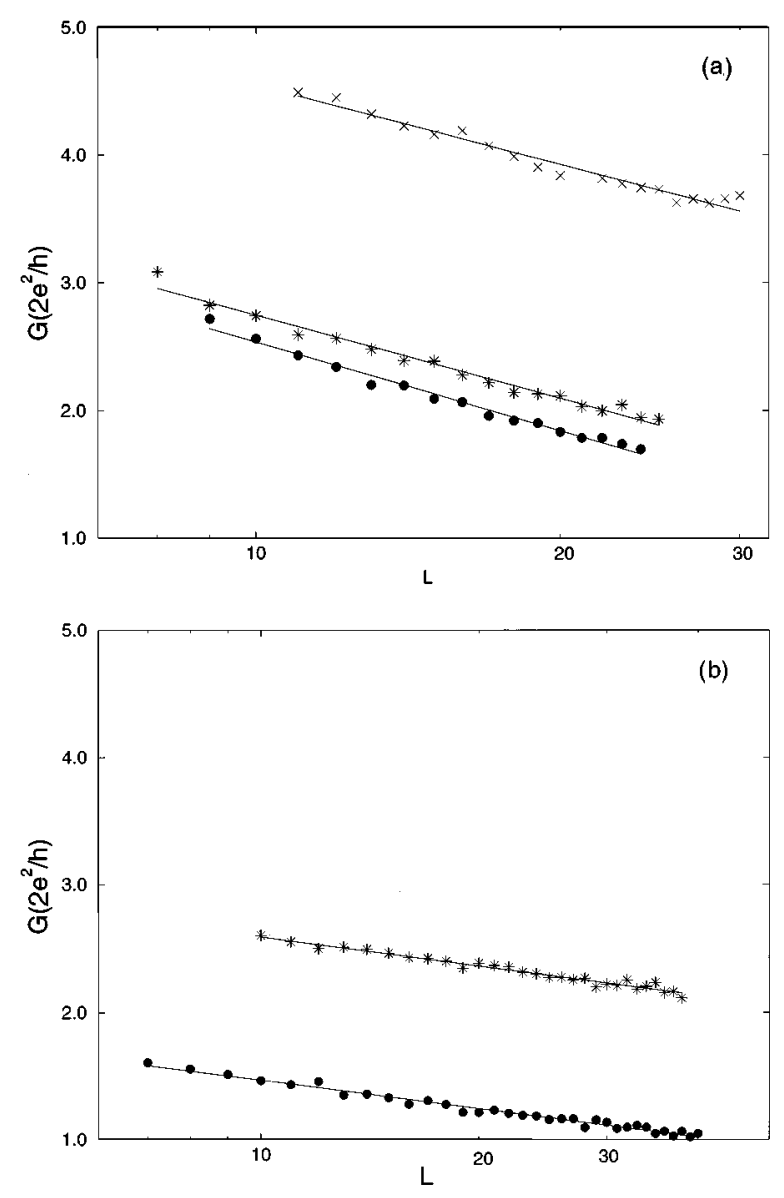

FIG. 1. Scaling results for 2D samples. (a): QP model for $P=0.84(\bigcirc), \quad P=0.85(*)$, and $P=0.90(\times)$. (b): Anderson model for $W=4$ (@) and $W=3(*)$. 1000-3000 configurations were used in the average. Numerical values of the slope are given in Tables I and II.

model. However, the value of $\alpha$ decreases to $2 / 3$ of its $2 \mathrm{D}$ value for multilayer samples, i.e., $\alpha=2$ when $M \geqslant 3$. Numerical results based on the Anderson model show that indeed $\alpha=1$ for the 2D case, as expected theoretically. However, a similar transition from $2 \mathrm{D}$ to multilayer samples occurs when $M$ is increased to 3 and beyond, i.e., $\alpha \sim 0.7$ for multilayer samples in the Anderson model. Thus a comparison between the QP model and the Anderson model shows both differences and similarities. The significantly different values of $\alpha$ could be due to the fact that the large contrast in $\epsilon_{i}$ in the QP model ( 0 and $\infty$ ) makes conventional perturbative scattering calculations inapplicable, thus putting the QP model in a different class from the Anderson model. On the other hand, the decrease in the $\alpha$ values when $M \geqslant 3$ is qualitatively understandable from the point of view that the scattering in the film-thickness direction tends to decrease the coherent backscattering correction. In the limit of a large film thickness $M$, the lowest-order correction to the conductivity along the film plane, $\sigma_{M \|}$, can be written as

$$
\sigma_{M \|}=\sigma_{0}-\frac{e^{2}}{\hbar} \frac{1}{2 \pi^{2} M} \int_{1 / L}^{1 / l} q d q \sum_{-N_{\max }}^{N_{\max }} \frac{1}{q^{2}+n^{2} \pi^{2} / M^{2}},
$$



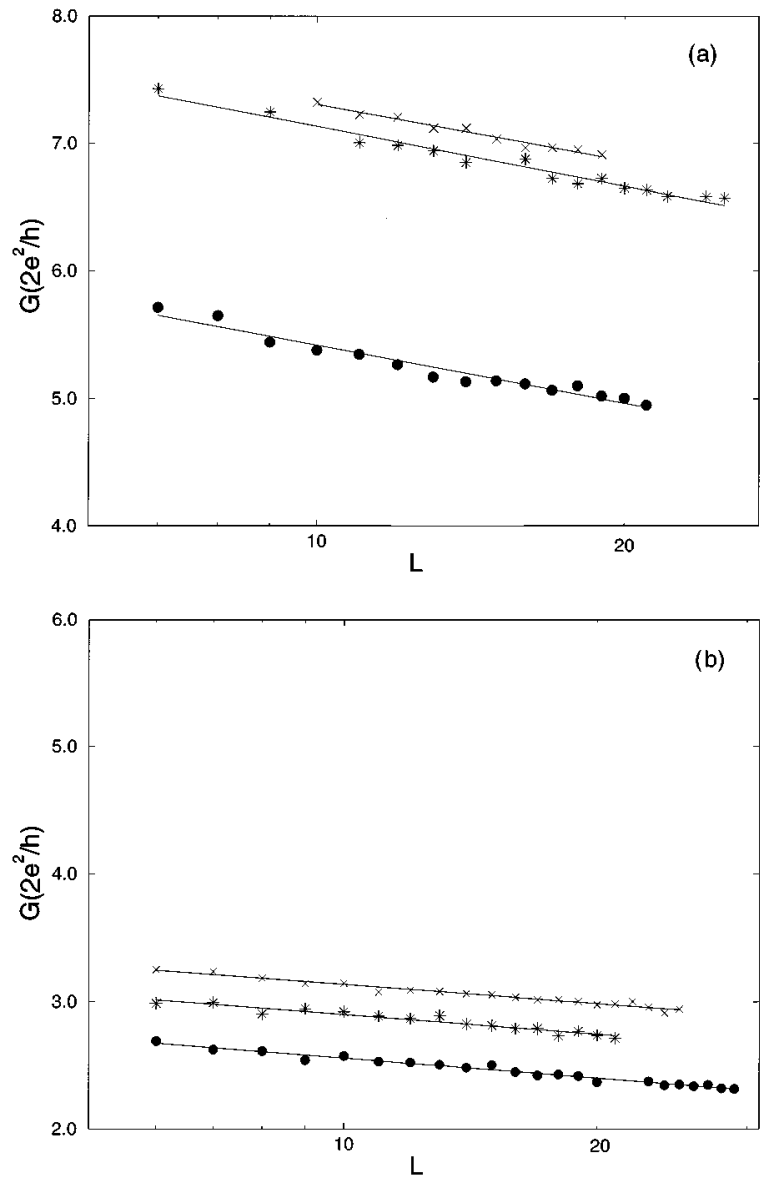

FIG. 2. (a) Scaling results for multilayer samples. (a): QP model for $M=3$ and $P=0.8(\bigcirc), M=3$ and $P=0.85(\times)$, and $M=4$ and $P=0.8(*)$. (b): Anderson model for $M=3$ and $W=5.5$ (○), $M=3$ and $W=5.0(\times)$, and $M=4$ and $W=6.0$ (*). 10003000 configurations were used in the average. Numerical values of the slope are given in Tables I and II.

where $N_{\max }$ in the summation is the same as the upper cutoff in the $q$ integration, $\sigma_{0}$ is the Drude conductivity (due to ladder diagrams), and $l$ and $L$ are the elastic mean free path and sample size along the film plane, respectively. It should be noted that Eq. (10) contains the implicit assumption that the film is sufficiently thick so that the electron transport in the vertical direction is diffusive as well. By performing the summation and the integration and retaining only the term that depends on $L$, we get the $\ln L$ dependence in the limit $L \gg M$ as

TABLE I. Numerical values of $\beta$ and $\alpha$ for the quantum percolation model.

\begin{tabular}{cccc}
\hline \hline No. of layers $(M)$ & $P$ & $\beta$ & $\alpha=\beta \pi$ \\
\hline 1 & 0.84 & $1.00 \pm 0.04$ & $3.1 \pm 0.1$ \\
1 & 0.85 & $0.94 \pm 0.04$ & $2.9 \pm 0.1$ \\
1 & 0.90 & $0.90 \pm 0.04$ & $2.8 \pm 0.1$ \\
3 & 0.80 & $0.65 \pm 0.03$ & $2.0 \pm 0.1$ \\
3 & 0.85 & $0.64 \pm 0.04$ & $2.0 \pm 0.1$ \\
4 & 0.80 & $0.68 \pm 0.03$ & $2.1 \pm 0.1$ \\
\hline \hline
\end{tabular}

TABLE II. Numerical values of $\beta$ and $\alpha$ for the Anderson model.

\begin{tabular}{cccc}
\hline \hline No. of layers $(M)$ & $W$ & $\beta$ & $\alpha=\beta \pi$ \\
\hline 1 & 3.0 & $0.33 \pm 0.01$ & $1.04 \pm 0.03$ \\
1 & 4.0 & $0.32 \pm 0.01$ & $1.01 \pm 0.03$ \\
3 & 5.0 & $0.22 \pm 0.01$ & $0.69 \pm 0.03$ \\
3 & 5.5 & $0.23 \pm 0.01$ & $0.72 \pm 0.03$ \\
4 & 6.0 & $0.22 \pm 0.02$ & $0.69 \pm 0.06$ \\
5 & 6.5 & $0.23 \pm 0.02$ & $0.72 \pm 0.06$ \\
\hline \hline & \\
\multicolumn{4}{c}{$\sigma_{M \|}=\sigma_{0}-\frac{e^{2}}{\hbar \pi^{2}} \frac{1}{2 M} \ln L}$,
\end{tabular}

and the scaling relation for the conductance, i.e., $G=\sigma M$, as

$$
G(L)=G_{0}-\frac{e^{2}}{\hbar \pi^{2}} \frac{1}{2} \ln L,
$$

which is independent of $M$. It should be noted that, due to the implicit assumption of diffusive transport in the vertical direction, $M$ cannot take the value of 1 in Eq. (11), i.e., Eq. (11) cannot be expected to agree with the $2 \mathrm{D}$ result by letting $M=1$. The factor $1 / 2$ in front of $\ln L$ on the right-hand side of Eq. (12) is due to the reduction in the probability of coherent backscattering in the limit when electron transport in the vertical direction is also fully diffusive. While our numerical results on multilayer samples do not indicate a full $1 / 2$ reduction in the value of $\alpha$, they are nevertheless smaller than that of the 2D samples. Hence the qualitative trend of our numerical results on multilayer samples can be explained by the weak-localization theory.

Since the QP model and the Anderson model give different values for $\alpha$, it follows that many prior experimental results may have to be reinterpreted in regard to their implications about the inelastic scattering mechanism(s). For example, for low-resistivity $\mathrm{Cu}$ films (50-500 $\AA$ ) at temperatures between 1 and $20 \mathrm{~K}$, Ref. 6 reported that $\alpha p=2$. By assuming $\alpha=1, p$ was assigned a value of 2 . Reference 10 also reported the value of $p=2$ in silver films. On the other hand, Ref. 7 reported that in high mobility MOSFET inversion layers at temperatures down to $0.05 \mathrm{~K}$, the independently measured $\alpha p$ and $\alpha$ values are $1 \pm 0.1$ and 1.0, respectively. Since inversion layers are intrinsically 2D systems, the $\alpha$ value agrees well with the Anderson model prediction. Thus $p=1$ may be deduced for these semiconductor samples.

In general, a value of $p=2$ is attributed to electronphonon scattering, ${ }^{8-10}$ whereas a value of $p=1$ is attributed to electron-electron scattering. ${ }^{7,11}$ If we now describe the elastic scattering in metallic films by the QP model and take the multilayer value of $\alpha$, i.e., $\alpha=2$, then the inelastic scattering mechanism in the two material systems, metallic films and MOSET inversion layers, are the same at low temperatures, i.e., electron-electron scattering with $p=1$.

In summary, our results on the QP model show that percolating metallic films follow a different $\ln L$ slope than that of the semiconducting films, thus demonstrating the nonuniversality of the $\alpha$ value. Moreover, we show that the value of $\alpha$ for finite-thickness films differs from the 2D case. When 
these differences are taken into account in the interpretation of experimental data, a different conclusion about the inelastic scattering mechanism is reached for metallic films. In view of our results, an experimental determination of $\alpha$ in low-resistivity metallic films would not only be interesting, but would also provide an independent verification of our conclusions.

One of us (Tan Li) wishes to thank Z. Q. Zhang for many helpful discussions. Tan Li was supported by research grant CERG HKUST612/95P.
${ }^{1}$ Ping Sheng, Introduction to Wave Scattering, Localization, and Mesoscopic Phenomena (Academic Press, Boston, 1995).

${ }^{2}$ P. A. Lee and D. S. Fisher, Phys. Rev. Lett. 47, 882 (1981).

${ }^{3}$ A. D. Stone, Phys. Rev. Lett. 54, 2692 (1985).

${ }^{4}$ Z. Q. Zhang and Ping Sheng, Phys. Rev. B 44, 3304 (1991).

${ }^{5}$ P. A. Lee and T. V. Ramakrishnan, Rev. Mod. Phys. 57, 287 (1985).

${ }^{6}$ L. Van den Dires, C. Van Haesendonck, and Y. Bruynseraede,
Phys. Rev. Lett. 46, 565 (1981).

${ }^{7}$ D. J. Bishop, D. C. Tsui, and R. C. Dynes, Phys. Rev. B 26, 773 (1982).

${ }^{8}$ G. Bergman, Z. Phys. B 48, 5 (1982).

${ }^{9}$ G. Bergman, Solid State Commun. 46, 347 (1983).

10 M. E. Gershenzon et al., Pis'ma Zh. Éksp. Teor. Fiz. 35, 467 (1982) [JETP Lett. 35, 576 (1982)].

${ }^{11}$ B. L. Al'tshuler and A. G. Aronov, Solid State Commun. 41, 33 (1982). 